\title{
Performance of urine lipoarabinomannan assays for paediatric tuberculosis in Tanzania
}

\author{
Inge Kroidl ${ }^{1,3,11}$, Petra Clowes ${ }^{3,11}$, Klaus Reither $^{4,5}$, Bariki Mtafya ${ }^{3}$, \\ Gabriel Rojas-Ponce ${ }^{3}$, Elias N. Ntinginya ${ }^{3}$, Mariam Kalomo ${ }^{6}$, \\ Lilian T. Minja $3,4,5,7$, Dickens Kowuor ${ }^{3}$, Elmar Saathoff ${ }^{1,2}$, Arne Kroidl $^{1,2,3}$, \\ Norbert Heinrich ${ }^{1,2}$, Leonard Maboko ${ }^{3}$, Matthew Bates ${ }^{8,9}, 10$, Justin O'Grady ${ }^{8,9,10,}$ \\ Alimuddin Zumla $8,9,10$, Michael Hoelscher ${ }^{1,2,3}$ and Andrea Rachow ${ }^{1,2,3}$
}

\begin{abstract}
Affiliations: ${ }^{1}$ Division of Infectious Diseases and Tropical Medicine, Medical Centre of the University of Munich (LMU), Germany. ${ }^{2}$ German Centre for Infection Research (DZIF), partner site Munich, Germany. ${ }^{3}$ National Institute for Medical Research-Mbeya Medical Research Centre, Mbeya, Tanzania. ${ }^{4}$ Medical Services and Diagnostic, Swiss Tropical and Public Health Institute, Basel, Switzerland. ${ }^{5}$ Medical Services and Diagnostic (Swiss TPH), University of Basel, Basel, Switzerland. 'Dept for Paediatrics and Child Health, Mbeya Referral Hospital, Mbeya, Tanzania. ${ }^{7}$ Ifakara Health Institute, Bagamoyo, Tanzania. ${ }^{8}$ University of Zambia-University College London Medical School Research and Training Project, University Teaching Hospital, Lusaka, Zambia. ${ }^{9}$ Division of Infection and Immunity, University College London, London, UK. ${ }^{10} \mathrm{NIHR}$ Biomedical Research Centre, University College London Hospitals, London, UK. ${ }^{11}$ Both authors contributed equally.
\end{abstract}

Correspondence: Inge Kroidl, Division of Infectious Diseases and Tropical Medicine, Medical Centre of the University of Munich (LMU), Germany. E-mail: ikroidlalrz.uni-muenchen.de

ABSTRACT We evaluated the diagnostic performance of two tests based on the release of lipoarabinomannan (LAM) into the urine, the MTB-LAM-ELISA assay and the Determine TB-LAM-strip assay, in children with suspected tuberculosis (TB) in a high TB/HIV-prevalence setting.

In a prospective study, 132 children with suspected active TB were assigned to diagnostic subgroups. Urine samples were subjected to testing by both assays to ascertain sensitivity and specificity. Host factors associated with positive LAM results were investigated and LAM excretion monitored after antituberculous treatment initiation.

$18(13.6 \%)$ children had culture-confirmed pulmonary TB. The assays' sensitivity was higher in HIVpositive versus HIV-negative children: 70\% (95\% confidence interval 35-93\%) versus 13\% (0-53\%) for MTB-LAM-ELISA and 50\% (19-81\%) versus 0\% (0-37\%) for Determine TB-LAM. In 35 (27\%) children with excluded active TB, both assays showed a specificity of $97.1 \%$ (85-100\%). Proteinuria and low body mass index were independently associated with LAM positivity. In most patients, LAM excretion declined to zero during or at conclusion of antituberculous treatment.

HIV/TB co-infected children might benefit from LAM-based tests to aid early TB diagnosis and subsequent positive impact on morbidity and mortality. Using LAM as a rule-in and treatment-monitoring tool may also show further potential.

@ERSpublications

Urine lipoarabinomannan assays show reasonable sensitivity in HIV+ but not HIV- TBinfected children http://ow.ly/N56aG

This article has supplementary data available from erj.ersjournals.com

Earn CME accreditation by answering questions about this article. You will find these at erj.ersjournals.com/misc/ cmeinfo.xhtml

Received: Jan 092015 | Accepted after revision: April 092015 | First published online: June 252015

Support statement: This study was supported by Bundesministerium für Bildung und Forschung and the European Commission as part of EuropAid. Funding information for this article has been deposited with FundRef.

Conflict of interest: None declared.

Copyright @ERS 2015 


\section{Introduction}

The diagnosis of tuberculosis (TB) is usually established by detecting mycobacteria, either through smear microscopy or culturing methods, which is considered the current gold standard. However, due to the paucibacillary nature of $\mathrm{TB}$ in children, the microbiological diagnosis is extremely difficult. Smear microscopy is positive in $<15 \%$ [1-3] and mycobacterial culture is positive in $20-80 \%$ of children with presumed TB $[1,2,4]$. Paediatric TB diagnosis is therefore mainly based on clinical assessments, scoring systems and radiological findings, which can be erroneous. In an autopsy study from Zambia, TB accounted for $18 \%$ of deaths in HIV co-infected and $26 \%$ of deaths in HIV-negative children with respiratory illnesses [5]. In contrast, during a recent review of the global burden of disease in children [6, 7], TB was not listed among the most common causes of paediatric deaths, demonstrating the low number of microbiologically confirmed cases.

For TB cases in clinical settings, the inconsistency between pathological and epidemiological data highlights the need for new and more accurate methods to diagnose TB in children, using non-invasive clinical samples. Lipoarabinomannan (LAM) detection in urine for TB diagnosis was first investigated in the late 1990s [8-10]. LAM is a $19 \mathrm{kD}( \pm 8.5 \mathrm{kD})$ lipopolysaccharide, specific to the cell wall of members of the Mycobacterium genus and is released from metabolically active or degrading bacterial cells [11, 12]. It can subsequently be detected in urine and other body fluids. Advantages of urine LAM diagnosis include the ease of specimen collection, short bench-time, low cost and relatively low training and set up requirements $[13,14]$. Ideally the LAM-strip assay can be even performed as a point-of-care test in remote settings. The reported sensitivity of the different LAM diagnostic tests in adults ranges between 13\% [15] and $67 \%$ [16], with best performance in patients with advanced HIV disease [15-18]. Data on children are scarce with only one study published previously on performance of LAM diagnostic tests in children [19].

The two diagnostic assays evaluated here, the MTB-LAM-ELISA (Chemogen, Portland, OR, USA) and the Determine TB-LAM (Alere, Waltham, MA, USA), have been studied recently in South African children, for whom they performed poorly [19]. We evaluated the diagnostic performance of both assays in Tanzanian children with presumed TB and a high HIV co-infection rate. We also investigated host factors that might be related to LAM performance, as well as the change in LAM excretion during the course of anti-TB treatment.

\section{Methods}

\section{Study design and setting}

We undertook a prospective observational study in children presenting with suspected TB at the outpatient department of the Mbeya Zonal Referral Hospital (Mbeya, Tanzania). The study was coordinated by the National Institute for Medical Research (NIMR) Mbeya Medical Research Centre (MMRC), in close collaboration with the Mbeya Zonal Referral Hospital. The study was approved by the ethics committee of the Tanzania National Institute for Medical Research and the local Mbeya Medical Research and Ethics Committee. Written informed consent for all children was obtained from an accompanying parent or a legal guardian. In addition, children aged $\geqslant 9$ years signed an assent form.

\section{Clinical study procedures}

From May 2008 till November 2010, we approached all children with suspected TB attending the outpatient clinic and invited them to take part in the study. Inclusion criteria were 6 weeks to 14 years of age, and at least one of the following symptoms: persistent unremitting cough for $>21$ days; repeated episodes of fever within the last 21 days; weight loss or failure to thrive within the previous 3 months. Children who had received antituberculous treatment within the last 3 months were excluded from the study. Recruitment procedures, baseline diagnostics, physical assessment and clinical treatment of this cohort have previously been described [20]. Follow-up visits were scheduled at 1, 3, 6 and 12 months after enrolment or after antituberculous treatment initiation. For this evaluation, children were retrospectively assigned to distinct diagnostic subgroups, based on the recently proposed classification by GraHAM et al. [21] (fig. 1).

A decision on antituberculous treatment initiation was made in liaison with the paediatric department of the Mbeya Zonal Referral Hospital and the District TB and Leprosy Coordinators and was based on microbiological and clinical findings (including tuberculin skin test and chest radiography), and previous medical history. Antituberculous treatment was administered following Tanzanian National Guidelines and patients diagnosed with HIV infection were referred for further staging and treatment to the relevant HIV Care and Treatment Centres.

\section{Sample collection and laboratory procedures}

Up to three induced sputum samples were collected from each child at baseline and processed for smear microscopy and Mycobacterium tuberculosis (MTB) culture [20]. Additionally, the Xpert MTB/rifampicin 


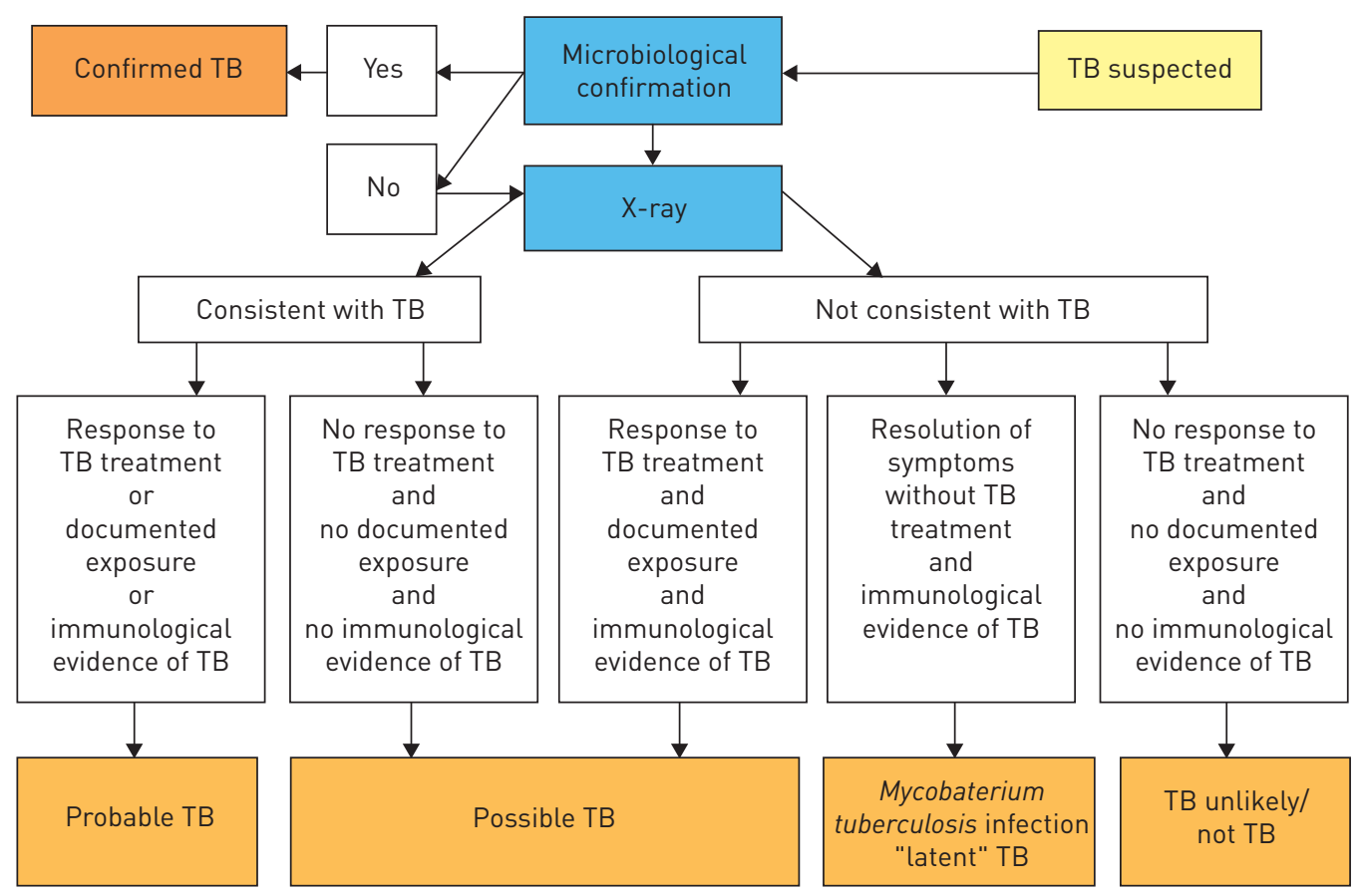

FIGURE 1 Algorithm for diagnostic classification. Diagnostic classification for children presenting with suspected pulmonary/intrathoracic tuberculosis (TB). In total, 379 sputum samples were analysed, 122 children gave three sputa, six children only two and three children only one sputum sample. One child classified as "No TB", gave no sputum sample. In five children with signs of extrapulmonary TB, one pleural fluid sample, one ascites sample and three lymph node aspirates were analysed. Mycobacterium tuberculosis was confirmed in the pleural fluid and in one lymph node aspirate. Both children were additionally diagnosed with sputum culture confirmed TB. All 132 children received chest radiography examination which was assessed by a radiologist and two clinical study investigators who were blinded to clinical and microbiological data. In case of discrepant readings a consensus radiography diagnosis was made after discussion among the investigators. Clinical follow-up of all analysed children allowed assessment of treatment response in those who received antituberculous treatment.

(RIF) assay (Cepheid, Sunnyvale, CA, USA) was performed on stored, frozen sputum samples as reported previously [20]. The results were not included in the diagnostic algorithm as all children were enrolled before Xpert endorsement by the World Health Organization (WHO). At each study visit, a urine sample was collected for LAM testing, either midstream urine when possible or with a collection bag in younger children. Within $8 \mathrm{~h}$ of collection, all urine was boiled at $95-100^{\circ} \mathrm{C}$ for $30 \mathrm{~min}$, centrifuged and the supernatants frozen at $-20^{\circ} \mathrm{C}$. For the execution of the MTB-LAM-ELISA assay, the thawed urine was processed in duplicate, according to the manufacturer's instructions as described previously [18]. For the Determine TB-LAM assay, $60 \mu \mathrm{L}$ of pre-treated urine was applied onto the sample pad of the LAM strip. After $25 \mathrm{~min}$, the test band colour intensity was compared to the colour intensity of a series of bands on a paper reference card supplied by the manufacturer. Grade 1 colour intensity and above were defined as positive results, consistent with the manufacturer's instructions at the time of the study [22]. According to the manufacturer (personal communication) and our own data, results for the Determine TB-LAM assay do not differ between pre-treated (boiled and centrifuged) or native urine samples. All lab staff performing LAM tests was blinded to culture results and clinical data. Furthermore, all urine samples underwent testing with urine dipsticks (Combur-Test; Roche, Basel, Switzerland) for detection of protein, glucose, leukocytes and erythrocytes.

All participants were screened for HIV, using the HIV1/2 STAT-PAK RDT (Chembio Diagnostics Systems, Medford, NY, USA) and following the manufacturer's instructions. RDT results were confirmed with a third generation ELISA (Biorad Laboratories, Redmond WA, USA) and, in case of discordance, retested by Western Blot (MPD HIV Blot 2.2, MP Biomedicals, Geneva, Switzerland). For children below the age of 2 years a PCR (Roche Amplicor) was performed instead of ELISA. In HIV-positive participants CD4 count and HIV viral load were determined by flow cytometry and PCR (Amplicor; Roche). HIV-positive children were classified according to the WHO classification of HIV-associated immunodeficiency in infants and children (see the online supplementary data) [23].

\section{Statistical analysis}

In contrast to the classification in the original study, all children were re-classified into five different diagnostic groups in order to comply with the recently published proposed consensus of paediatric clinical 
case definition by Graham et al. [21]. Statistical analyses were performed using Stata statistics software (version 12; Stata Corp., College Station, TX, USA). The sensitivity, specificity and their respective confidence intervals were calculated using the "diagt" command in Stata. Pearson's Chi-squared test was used to compare binominal variables between groups (confirmed TB versus no-TB or HIV-positive versus HIV-negative) and the non-parametric Wilcoxon rank sum test was used to compare selected baseline characteristics of continuous variables, since none of the continuous variables was normally distributed. The correlation of optical density values and grading of MTB-LAM ELISA and Determine TB-LAM assays were compared by Spearman rank correlation. Univariable and multivariable log link binomial regression analyses, using robust variance estimates, were performed to examine the influence of potentially important factors on LAM positivity.

\section{Results}

Between May 2008 and November 2010, 180 children with presumed intrathoracic TB were enrolled into the study. Due to an incomplete data set, 48 study subjects were excluded from this analysis. In the majority of excluded children, 37 (77\%) out of 48, no urine sample was collected at baseline. As depicted in figure 2, all diagnostic groups were equally affected by this exclusion criterion. The remaining 132 children were assigned to one of five distinct diagnostic classification groups in line with the definitions by Graham et al. [21] (figs. 1 and 2). Antituberculous treatment was offered to all children with confirmed and probable $\mathrm{TB}$ and to more than half of the children with possible TB, depending on their clinical and radiological presentation. Five children initially received antituberculous treatment, but a different diagnosis was later established. $14(10.5 \%)$ children who demonstrated immunological evidence of TB at baseline, but improved without antituberculous treatment, were classified with MTB infection. Overall, a decision for antituberculous treatment was made for $80(61 \%)$ of the 132 children. 69 of all recruited

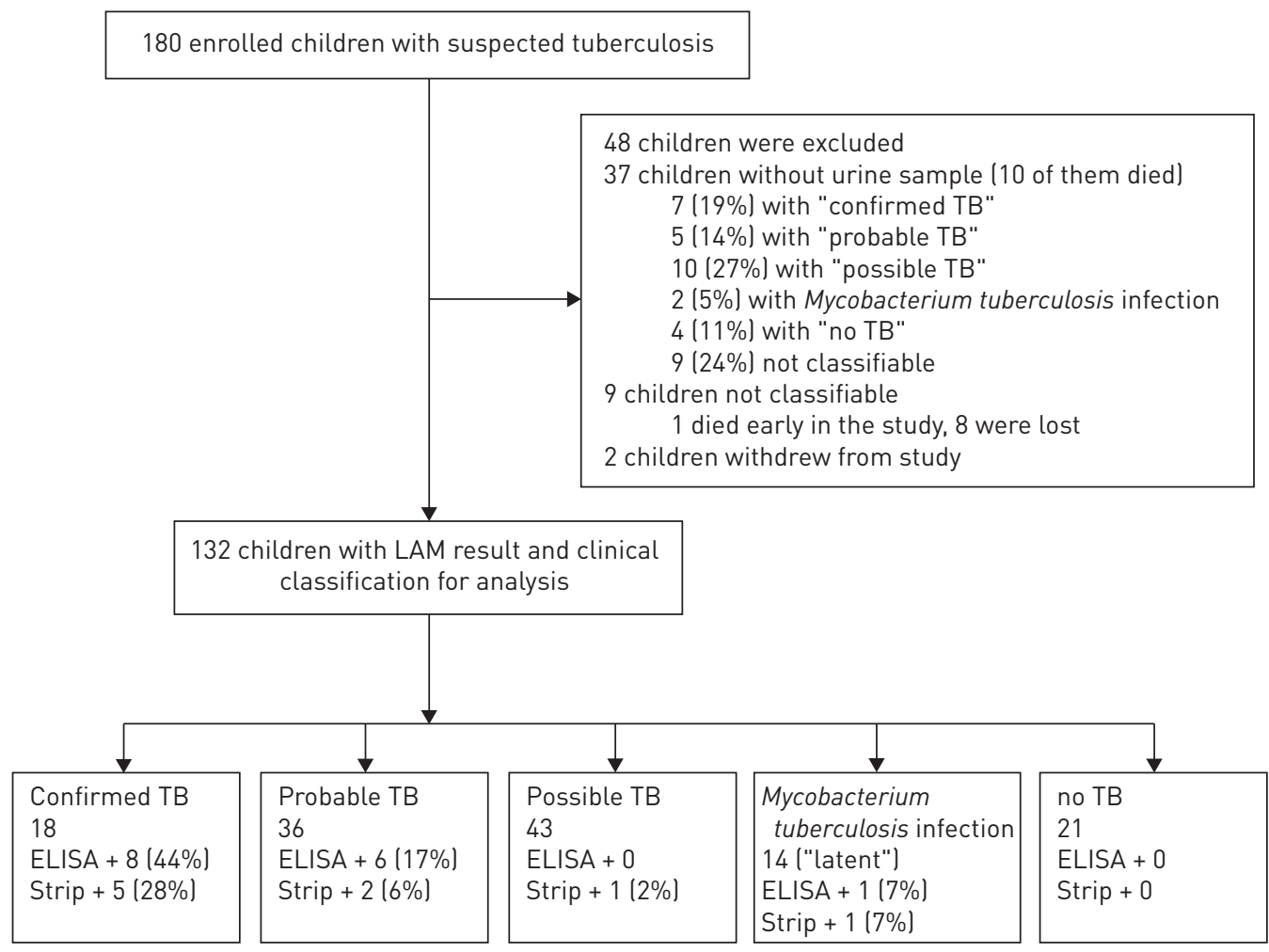

FIGURE 2 Study flow chart with diagnostic classification of participants. The number of Mycobacterium tuberculosis-lipoarabinomannan (LAM)-ELISA positive urines (ELISA +) and the number of Determine TB LAM-Strip positive urines $($ STRIP + ) is given for each clinical diagnostic group. Confirmed TB: 18 children had microbiological confirmation of M. tuberculosis infection. Probable TB: tuberculosis could not be microbiologically confirmed, but was highly probable in 36 children and antituberculous treatment was offered to all of them. Possible TB: tuberculosis could not be reliably excluded in 43 children, but they did not fulfil criteria for any other group 24 (56\%) of the children with possible tuberculosis received tuberculosis treatment. MTB-infection: 14 children demonstrated immunologic evidence of TB, but improved without antituberculous treatment. None of them died. No TB: In 21 children, tuberculosis was retrospectively reliably excluded. Five children initially received antituberculous treatment, but subsequently an alternative diagnosis was established. The tuberculin skin test was non-reactive in all children, all recovered during the follow-up. 
children were hospitalised at or during enrolment, 42 of whom were included in this analysis. Demographical, radiological and clinical data of all children and differences in parameters per group at the time of enrolment are displayed in table 1. Apart from the clinical and radiological data presented in table 1 , no further data on extra-thoracic TB disease spectrum were systematically collected.

Out of the group of culture-confirmed TB, eight TB-cases were identified by the MTB-LAM-ELISA and five by the Determine TB-LAM-strip assay, resulting in a sensitivity of 44\% (95\% CI 22-69\%) and 28\% (95\% CI 10-54\%), respectively (table 2). Among the children with a strong clinical suspicion of $\mathrm{TB}$, six and two (additional) children were flagged by the MTB-LAM-ELISA and the Determine TB-LAM-strip assay, respectively. In a combined approach, including children of both groups, the sensitivity was $26 \%$ (95\% CI $15-$ $40 \%)$ and $13 \%$ (95\% CI 5-25\%), respectively. Concerning specificity, none of the LAM-assays was positive in the group of children where TB was reliably excluded, resulting in a specificity of $100 \%$ (95\% CI $84-100 \%)$ at baseline evaluation. One child with MTB infection had positive LAM diagnostic tests. If this diagnostic group is included in the calculation, the overall specificity was $97.1 \%$ (95\% CI 85-100\%) for both tests. In the direct comparison of both LAM-assays, the MTB-LAM-ELISA detected 14 of the 54 children with confirmed or probable TB, whereas the Determine TB-LAM-strip assay only identified 7 of those children.

The overall HIV prevalence in our study cohort was 51\%. In children with confirmed TB, the sensitivity of both LAM diagnostic tests was significantly higher in HIV-positive compared with HIV-negative children: $70 \%$ (95\% CI 35-93\%) versus $13 \%$ (95\% CI 0-53\%) were detected by the MTB-LAM-ELISA and 50\% (95\% CI 19-81\%) versus 0\% (95\% CI 0-37\%) by the Determine TB-LAM assay (table 2). The comparison of the performance of both LAM-assays in children with advanced or severe immunosuppression versus those with mild or no immunosuppression does however not suggest a higher sensitivity of LAM diagnostics in those with advanced HIV infection.

Employing binominal regression analysis, we found an independent and strong association between proteinuria and LAM positivity for the MTB-LAM-ELISA (table 3). No other urine-associated factors such as haematuria, leukocyturia, specific weight or glycosuria could be linked with a positive LAM result (data not shown). Testing the influence of additional host factors, we found that a positive MTB-LAM-ELISA was independently associated with a low body mass index (BMI) for age and with higher mortality (table 3).

Similarly, a significant association of the Determine TB-LAM with low BMI was found. Trends for increased risk were demonstrated for concomitant HIV infection and proteinuria, but did not reach significance (table 4). No significant influence of age or sex on test positivity could be demonstrated for either assay.

In the per-sample analysis of the 16 urine samples identified as positive by one of the assays at baseline, the quantitative readouts of the Determine TB-LAM (grade 1-5) and the LAM ELISA optical density measurements showed a good correlation, reflected by a Spearman rank correlation of $r h o=0.79, \mathrm{p}=0.0003$ (data not shown). Seven out of eight positive Determine TB-LAM tests were graded 2 or above, the remaining test was grade 1 , which was a positive result at the time of testing.

In 13 out of 14 children with confirmed or probable TB and a positive LAM result at baseline, both LAM assays were performed at follow up visits. Figure 3 shows the general decline of signal positivity for the MTB-LAM-ELISA at different time points after starting antituberculous treatment. Overall, signal intensity reached zero after 3 months of antituberculous treatment in six participants with MTB-LAM-ELISA-positive urine samples at baseline. Only one child excreted measurable LAM more than 7 month after antituberculous treatment started. Clinically, all children responded well to treatment and were considered cured after 6 months of therapy. The same trend towards a major decline of signal positivity during antituberculous treatment was seen for the Determine TB LAM assay (data not shown).

In the HIV-infected subgroup with confirmed TB diagnosis, both LAM-diagnostic tests demonstrated a better sensitivity than smear microscopy, which detected only $30 \%$ of all HIV-positive confirmed TB-cases (fig. 4). The combination of smear microscopy and Determine TB LAM strip led to a combined sensitivity of $60 \%$ (fig. 4) and smear microscopy plus MTB-LAM-ELISA amounted to a sensitivity of $80 \%$. Combining the Xpert MTB/RIF assay and any of the LAM assays led to an overall sensitivity of $90 \%$ amongst these children, as both LAM-tests detected one confirmed TB case which was negative in the Xpert MTB/RIF-assay (fig. 4).

\section{Discussion}

We evaluated the diagnostic performance of the MTB-LAM-ELISA and the new and easier-to-use Determine TB-LAM strip test in a paediatric cohort from a resource limited setting in Tanzania with high TB and HIV burden. In line with studies on adults $[15,16,18,24]$, both assays showed a poor sensitivity when compared to MTB culture, which increased significantly in children with HIV co-infection. However, contrary to reports on adults $[15,16,25]$, a positive correlation between advanced immunosuppression and increased sensitivity of the LAM-tests could not be confirmed by our paediatric data. Interestingly, LAWN et al. [26] reported that 
TABLE 1 Baseline characteristics of children in different diagnostic classes

\begin{tabular}{|c|c|c|c|c|c|c|c|c|}
\hline & Included children & Excluded children ${ }^{\#}$ & Confirmed TB & Probable TB & Possible TB & MTB infection & No TB & p-value \\
\hline Subjects $\mathrm{n}$ & 132 & 45 & 18 & 36 & 43 & 14 & 21 & \\
\hline Sex male ${ }^{+}$ & $70(53)$ & $22(50)$ & 7 (39) & $19(53)$ & $22(51)$ & $8(57)$ & $14(67)$ & 0.083 \\
\hline Age years & $6.8(3.9-9.5)$ & $2.1(0.7-5.5)$ & $7.3(4.8-11.5)$ & $6.8(3.9-9.4)$ & $7.2(3.9-10.0)$ & $5.2(2.6-8-8)$ & $5.9(3.9-10.1)$ & 0.383 \\
\hline BMI for age z-score & $-0.44(-1.5-0.4)$ & $-1.35(-3.1-0.1)$ & $-1.21(-2.6-0.1)$ & $-0.11(-1.2-0.6)$ & $-0.96(-2.0-0.2)$ & $0.56(-0.3-1.1)$ & $-0.49(-1.2-0.4)$ & 0.105 \\
\hline TST reactive & $44(36)$ & $11(31)$ & $10(59)$ & $18(55)$ & $5(12)$ & $11(92)$ & 0 & $<0.001$ \\
\hline Proteinuria $>30 \mathrm{mg}^{\S}$ & $13(10)$ & $3(20)$ & $4(22)$ & $4(11)$ & $4(10)$ & 0 & $1(5)$ & 0.104 \\
\hline Mortality & $11(8)$ & $11(24)$ & 0 & $3(8)$ & $8(19)$ & 0 & 0 & \\
\hline Days to treatment & $21(7-58)$ & $15(5-40)$ & $9(6-25)$ & $18(7-44)$ & $31(13-76)$ & $\mathrm{N} / \mathrm{A}$ & $40(6-61)$ & 0.307 \\
\hline HIV-positive & $67(51)$ & $19(53)$ & $10(56)$ & $18(50)$ & $22(51)$ & $4(29)$ & $13(62)$ & 0.688 \\
\hline \multicolumn{9}{|l|}{ In HIV-positive children: } \\
\hline ART at baseline & $17(25)$ & $3(16)$ & $1(10)$ & $5(28)$ & $7(32)$ & 0 & $4(31)$ & 0.231 \\
\hline No significant immunosuppression ${ }^{f}$ & $18(27)$ & $1(6)$ & $1(10)$ & $5(28)$ & $5(24)$ & $1(25)$ & $6(46)$ & 0.062 \\
\hline Mild immunosuppression ${ }^{f}$ & $5(8)$ & $1(6)$ & $2(20)$ & 0 & $2(10)$ & 0 & $1(8)$ & 0.385 \\
\hline Advanced immunosuppression ${ }^{f}$ & $8(12)$ & 0 & $3(30)$ & $2(11)$ & $1(5 \%)$ & $1(25)$ & $1(8)$ & 0.162 \\
\hline Severe immunosuppression ${ }^{f}$ & 35 (53) & 15 (88) & $4(40)$ & $11(61)$ & $13(62)$ & $2(50)$ & 5 (38) & 0.940 \\
\hline
\end{tabular}

Data are presented as $\mathrm{n}(\%)$ or median (interquartile range), unless otherwise stated. Site of tuberculosis for children with confirmed tuberculosis (TB): two perihilar infiltrate, nine hilar lymphadenopathy, three tuberculous bronchopneumonia, four tuberculous pleural effusion. Site of tuberculosis for children with probable TB: nine perihilar infiltrate, nine hilar lymphadenopathy, seven tuberculous bronchopneumonia, three tuberculous pleural effusion, three military TB, one cavitating pulmonary TB. MTB: Mycobacterium tuberculosis; BMI: body mass index: TST, tuberculin skin test; ART: antiretroviral therapy. \#: for three of the 48 excluded children no further clinical data were available; ${ }^{\text {I: }} \mathrm{p}$-value for comparison between children with confirmed TB and no TB using Pearson's Chi-squared test for binary and the Wilcoxon rank-sum test for continuous variables; ${ }^{+}$: for one (excluded) child no sex information was available; ${ }^{\S}$ : for two included children no urine dipstick result was available; ${ }^{f}$ : for one included child no CD4 count was measured and the level of immunosuppression could not be calculated. 
TABLE 2 Diagnostic performance of both lipoarabinomannan (LAM) assays

Sensitivity

Confirmed TB Probable TB Combined TB diagnosis
Specificity"

TB excluded Combined TB excluded and TB infection

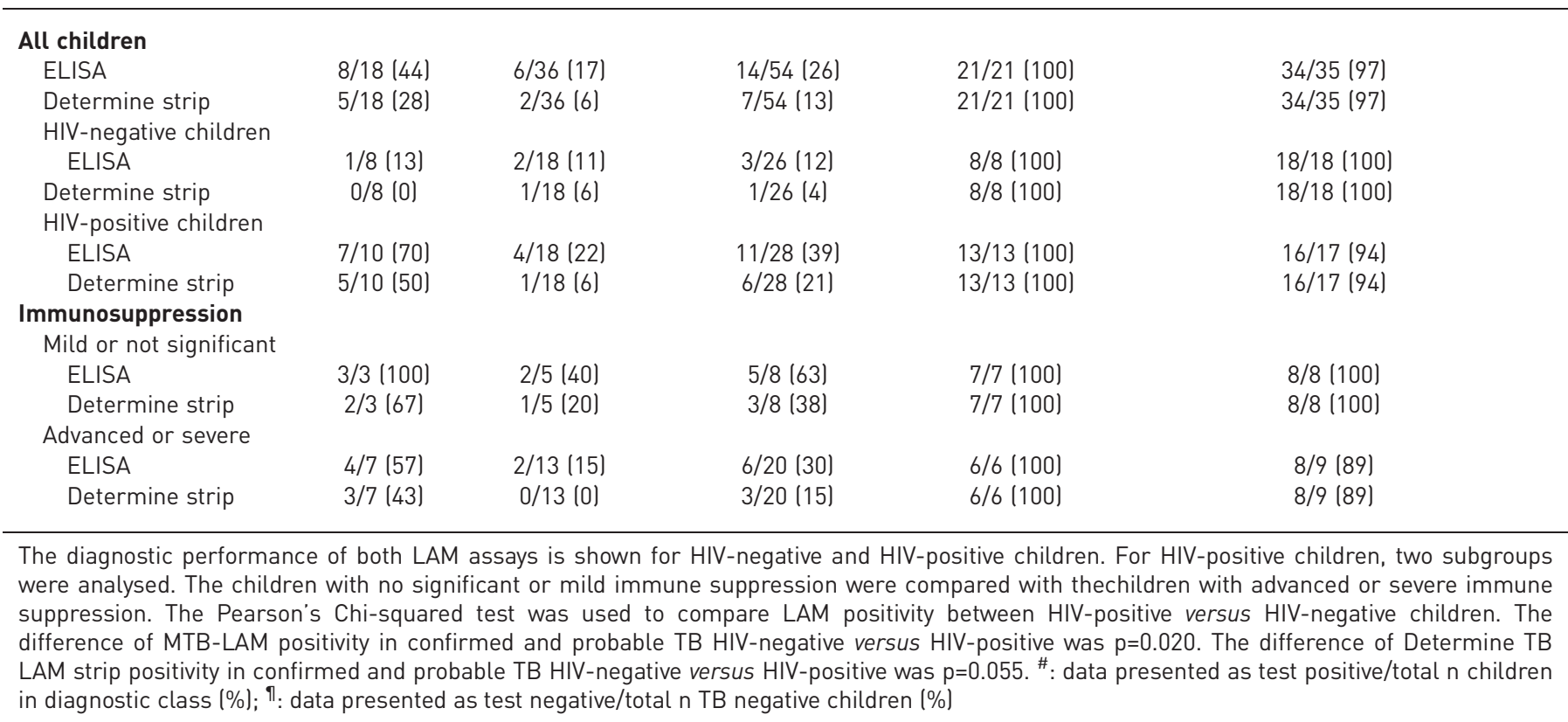

\begin{tabular}{|c|c|c|c|c|}
\hline & Subjects & LAM & $\operatorname{RR}(95 \% \mathrm{CI})$ & p-value \\
\hline Age & 54 & $14(26)$ & $1.07(0.93-1.22)$ & 0.371 \\
\hline \multicolumn{5}{|l|}{ Sex } \\
\hline Female & 28 & $8(29)$ & 1 & \\
\hline Male & 26 & $6(23)$ & $0.81(0.32-2.03)$ & 0.650 \\
\hline \multicolumn{5}{|l|}{ HIV status } \\
\hline Negative & 26 & $3(12)$ & 1 & \\
\hline Positive & 28 & 11 (39) & $3.40(1.06-11.0)$ & 0.040 \\
\hline \multicolumn{5}{|l|}{ Immunosuppression } \\
\hline None or mild & 8 & 5 (63) & 1 & \\
\hline Advanced or severe & 20 & $6(30)$ & $0.48(0.20-1.15)$ & 0.100 \\
\hline \multicolumn{5}{|l|}{ Proteinuria } \\
\hline No & 39 & 7 (18) & 1 & \\
\hline Yes & 14 & $7(50)$ & $2.79(1.18-6.58)$ & 0.019 \\
\hline \multicolumn{5}{|l|}{ BMI for age } \\
\hline Z score & 54 & $14(26)$ & $0.76(0.60-0.96)$ & 0.020 \\
\hline \multicolumn{5}{|l|}{ Died } \\
\hline No & 51 & $12(24)$ & 1 & \\
\hline Yes & 3 & $2(67)$ & $2.83(1.09-7.32)$ & 0.032 \\
\hline \multicolumn{5}{|l|}{ TB classification } \\
\hline Confirmed TB & 18 & $8(44)$ & 1 & \\
\hline Probable TB & 36 & $6(17)$ & $0.38(0.15-0.93)$ & 0.033 \\
\hline \multicolumn{5}{|c|}{$\begin{array}{l}\text { Data are presented as } \mathrm{n} \text { or } \mathrm{n}(\%) \text {, unless otherwise specified. Results from separate univariable binomial } \\
\text { log link regression models for each of the above variables. A significantly increased risk for a positive } \\
\text { Mycobacterium tuberculosis (MTB)- lipoarabinomannan (LAM)-ELISA result was found in participants with } \\
\text { confirmed tuberculosis (TB) diagnosis, concomitant HIV infection, proteinuria, low body mass index (BMI) } \\
\text { and participants who died during the course of the trial. In a multivariable model, which only included HIV, } \\
\text { BMI and proteinuria, the risk ratios (RRs) and p-values remained similar, demonstrating an independent } \\
\text { association of these variables with LAM-positivity. }\end{array}$} \\
\hline
\end{tabular}




\begin{tabular}{|c|c|c|c|c|}
\hline & Subjects & LAM & RR (95\% CI) & p-value \\
\hline Age & 54 & $7(13)$ & $1.23(0.96-1.58)$ & 0.100 \\
\hline \multicolumn{5}{|l|}{ Sex } \\
\hline Female & 28 & $5(18)$ & 1 & \\
\hline Male & 26 & $2(8)$ & $0.43(0.09-2.06)$ & 0.292 \\
\hline \multicolumn{5}{|l|}{ HIV status } \\
\hline Negative & 26 & $1(4)$ & 1 & \\
\hline Positive & 28 & $6(21)$ & $5.57(0.70-44.1)$ & 0.104 \\
\hline \multicolumn{5}{|l|}{ Immunosuppression } \\
\hline None or mild & 8 & 3 (38) & 1 & \\
\hline Advanced or severe & 20 & $3(15)$ & $0.40(0.09-1.62)$ & 0.199 \\
\hline \multicolumn{5}{|l|}{ Proteinuria } \\
\hline No & 39 & $4(10)$ & 1 & \\
\hline Yes & 14 & $3(21)$ & $2.09(0.53-8.30)$ & 0.295 \\
\hline \multicolumn{5}{|l|}{ BMI for age } \\
\hline Z score & 54 & $7(13)$ & $0.67(0.48-0.92)$ & 0.012 \\
\hline \multicolumn{5}{|l|}{ Died } \\
\hline No & 51 & $6(12)$ & 1 & \\
\hline Yes & 3 & $1(33)$ & $2.83(0.48-16.9)$ & 0.253 \\
\hline \multicolumn{5}{|l|}{ TB classification } \\
\hline Confirmed TB & 18 & $5(28)$ & 1 & \\
\hline Probable TB & 36 & $2(6)$ & $0.20(0.04-0.95)$ & 0.042 \\
\hline
\end{tabular}

Data are presented as $\mathrm{n}$ or $\mathrm{n}(\%)$, unless otherwise stated. Results from separate univariable binomial log link regression models for each of the above variables. A significantly increased risk for a positive Determine TB LAM was demonstrated for confirmed tuberculosis (TB) diagnosis and low body mass index (BMI) for age, similar to the association demonstrated for the Mycobacterium tuberculosis (MTB)lipoarabinomannan (LAM)-ELISA. Trends for increased risk were demonstrated for concomitant HIV infection and proteinuria, but did not reach significance level.

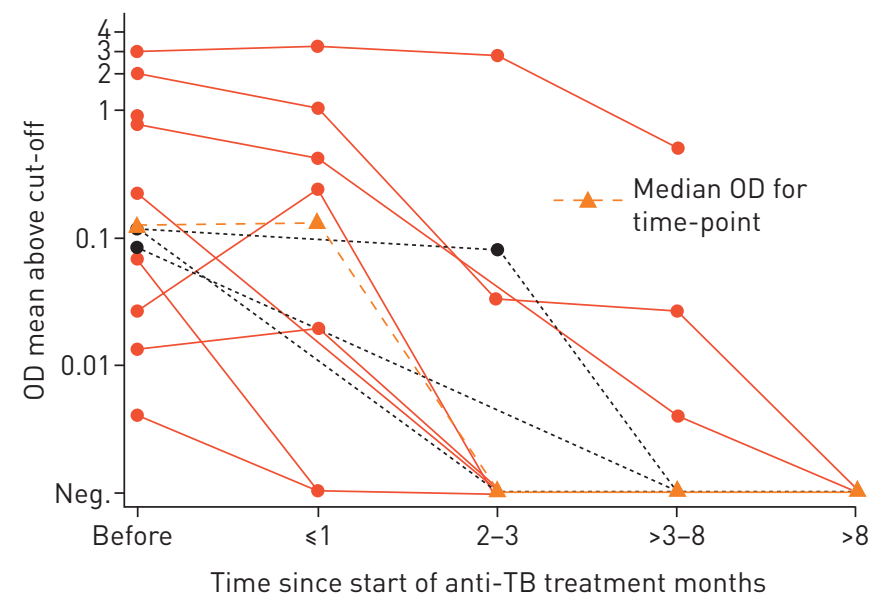

FIGURE 3 Signal intensity of Mycobacterium tuberculosis (MTB)- lipoarabinomannan (LAM)-ELISA at different time points after initiation of tuberculosis (TB) treatment. A drop of signal intensity of the MTB-LAM-ELISA for children with confirmed and probable TB during the course of antituberculous treatment was observed. Optical density (OD) results for HIV-positive participants are shown in red solid lines, results for HIV-negative participants as black dashed lines. The median OD at baseline was higher for HIV-positive children compared with HIV-negative children ( 0.221 versus 0.118 , respectively). The signal intensity reached 0 after 3 month of treatment in six participants. One child excreted measurable LAM more than 7 months after TB therapy started. Y-axis shows logarithmic scale for mean OD. Neg.: negative.

especially those (adult) individuals with advanced disease and poor outcome were detected by urine-based TB diagnostic assays such as the LAM-test. The fact that, in our study, LAM positivity was positively and independently associated with culture-confirmed TB, a low BMI-Z-score and death might further support these findings. Furthermore, and equally to findings from adult studies [18], we found a correlation between 


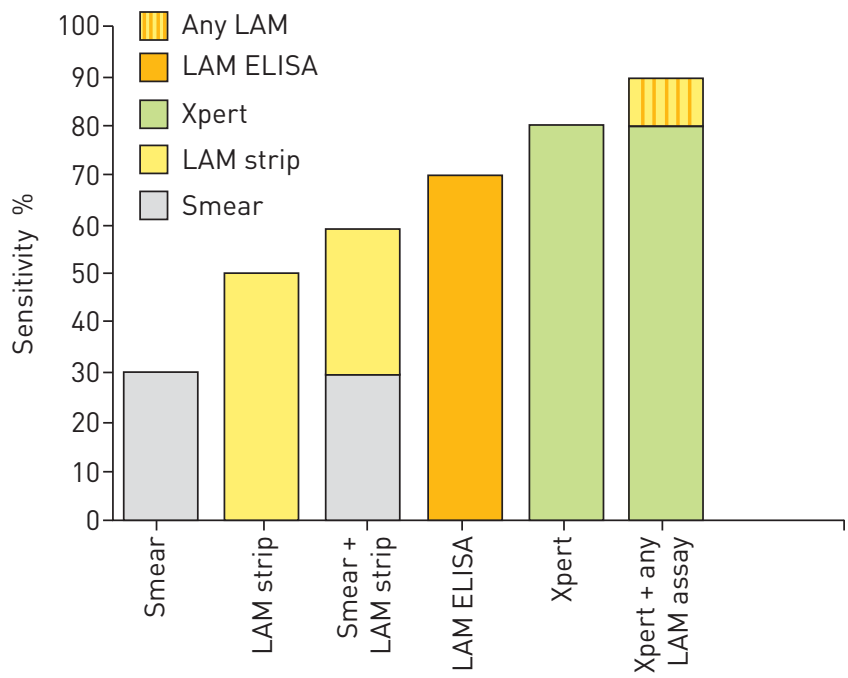

FIGURE 4 Sensitivity of single and combined tuberculosis (TB) diagnostic tests in HIV-infected children. This graph shows the sensitivity of each diagnostic test alone and the additional gain when combining lipoarabinomannan (LAM) diagnostic tests with either smear microscopy or Xpert MTB/RIF-assay in HIV-postive individuals with confirmed TB. LAM-Strip: Determine-TB-LAM; LAM ELISA: MTB LAM-ELISA; Xpert: GeneXpert.

proteinuria and LAM-positivity, indicating that the excretion of LAM might also depend on the condition of the kidney membrane. This and other risk factors for LAM-positivity require further investigation in order to better define the potential paediatric target group for LAM-based diagnostics in the future.

Our data regarding the overall sensitivity of the Determine TB-LAM strip test, were comparable with those previously published for a paediatric cohort from Cape Town [19]. However, the Cape Town study was unable to demonstrate an improved LAM sensitivity in TB/HIV co-infected children. Unfortunately, an in-depth comparison of our findings with those of NICOL et al. [19] is hampered by the extremely low sensitivity of both LAM tests, as well as the poor correlation between the MTB-LAM-ELISA and the Determine TB-LAM strip test in that study.

Contrary to our previously published data from the same setting in Tanzania $[18,27]$ and the data published by NiCOL et al. [19], the specificity of both LAM assays was high in our cohort. Depending on the hygienic standards and the procedures during sample collection, false-positive LAM results had been observed previously, most likely due to contamination of the sample with environmental mycobacteria or other bacteria [27]. In this study, we collected urine samples with great precautions, including washing instructions and the use of clean containers, in order to avoid false positive results. However, especially in HIV-positive children response to antituberculous treatment should be closely monitored as LAM-based urine tests cross react with other pathogenic mycobacteria (e.g. Mycobacterium avium complex) and might influence results. Although the sensitivity of both LAM assays was unsatisfactory, and the requirements for correct sample collection are high, our data indicate that the use of urine LAM-based tests as rule-in test could still be advantageous for children in certain settings where sophisticated TB diagnostics are not available but strict urine collection criteria can be adhered to.

Furthermore, the fact that a decline of LAM-excretion could be measured during treatment may open up a possibility to monitor antituberculous treatment success in LAM positive children. Although LAM-based TB diagnosis has the disadvantage that it does not include information on drug resistance, it could be hypothesised that ongoing excretion of urine LAM during treatment might provide information on insufficiently treated drug resistant TB. Larger studies with a long clinical follow up are needed to further scrutinise this hypothesis.

One weakness of our study is the relatively low number of 18 confirmed TB cases, which prevented a definite conclusion of the performance of LAM assays in certain subgroups, such as HIV-negative children or in children with HIV co-infection and different levels of immunosuppression. Furthermore, the exclusion of 37 children from the analysis because no urine sample or LAM result was available at baseline, may have introduced a selection bias. Urine collection was more cumbersome especially in younger and sicker children as it requires both the child's and the caregiver's cooperation and may be affected by medical causes such as dehydration. However, gathering a urine specimen was not a priority when the main study was designed, and we are confident that the proportion of children with an available 
sample would be higher if staff could be trained accordingly. We hypothesise that the exclusion of young children with advanced disease might have led rather to an underestimation of LAM-sensitivity, as data from our analysis indicate.

In conclusion, both LAM tests demonstrated a reasonable sensitivity in HIV-positive TB-infected children, whereas for HIV-negative children the sensitivity was extremely poor. The combination of LAM tests with other rapid TB diagnostics could substantially improve the detection of TB in HIV co-infected children. This holds promise for earlier TB-diagnosis in children, which might in turn have an impact on childhood morbidity and mortality associated with TB. Additionally, clean sample collection methods to achieve a high specificity have to be defined in more detail.

\section{Acknowledgements}

We thank the staff at the Active Detection of Active Tuberculosis (ADAT) paediatric TB clinic, the laboratories at NIMR-MMRC (Mbeya, Tanzania) and staff from the paediatric wards at the Mbeya Zonal Referral Hospital for their dedicated work, as well as the children and their parents or guardians who agreed to participate in this study. Also we want to thank Alere (Waltham, MA, USA) for providing the Determine TB-LAM Strips and some MTB-LAM-ELISA.

\section{References}

Marais BJ, Pai M. Recent advances in the diagnosis of childhood tuberculosis. Arch Dis Child 2007; 92: 446-452.

Starke JR. Pediatric tuberculosis: time for a new approach. Tuberculosis (Edinb) 2003; 83: 208-212.

Zar HJ, Hanslo D, Apolles P, et al. Induced sputum versus gastric lavage for microbiological confirmation of pulmonary tuberculosis in infants and young children: a prospective study. Lancet 2005; 365: 130-134.

4 Marais BJ, Hesseling AC, Gie RP, et al. The bacteriologic yield in children with intrathoracic tuberculosis. Clin Infect Dis 2006; 42: e69-e71.

5 Chintu C, Mudenda V, Lucas S, et al. Lung diseases at necropsy in African children dying from respiratory illnesses: a descriptive necropsy study. Lancet 2002; 360: 985-990.

6 Liu L, Johnson HL, Cousens S, et al. Global, regional, and national causes of child mortality: an updated systematic analysis for 2010 with time trends since 2000. Lancet 2012; 379: 2151-2161.

7 Liu L, Oza S, Hogan D, et al. Global, regional, and national causes of child mortality in 2000-13, with projections to inform post-2015 priorities: an updated systematic analysis. Lancet 2015; 385: 430-440.

8 Hamasur B, Bruchfeld J, Haile M, et al. Rapid diagnosis of tuberculosis by detection of mycobacterial lipoarabinomannan in urine. J Microbiol Methods 2001; 45: 41-52.

9 Hamasur B, Kallenius G, Svenson SB. A new rapid and simple method for large-scale purification of mycobacterial lipoarabinomannan. FEMS Immunol Med Microbiol 1999; 24: 11-17.

10 Tessema TA, Hamasur B, Bjun G, et al. Diagnostic evaluation of urinary lipoarabinomannan at an Ethiopian tuberculosis centre. Scand J Infect Dis 2001; 33: 279-284.

11 Boehme C, Molokova E, Minja F, et al. Detection of mycobacterial lipoarabinomannan with an antigen-capture ELISA in unprocessed urine of Tanzanian patients with suspected tuberculosis. Trans R Soc Trop Med Hyg 2005; 99: 893-900.

12 Chan J, Fan XD, Hunter SW, et al. Lipoarabinomannan, a possible virulence factor involved in persistence of Mycobacterium tuberculosis within macrophages. Infect Immun 1991; 59: 1755-1761.

13 Peter JG, Cashmore TJ, Meldau R, et al. Diagnostic accuracy of induced sputum LAM ELISA for tuberculosis diagnosis in sputum-scarce patients. Int J Tuberc Lung Dis 2012; 16: 1108-1112.

14 Peter JG, Theron G, Dheda K. Can Point-of-Care Urine LAM Strip Testing for Tuberculosis Add Value to Clinical Decision Making in Hospitalised HIV-Infected Persons? PLoS One 2013; 8: e54875.

15 Dheda K, Davids V, Lenders L, et al. Clinical utility of a commercial LAM-ELISA assay for TB diagnosis in HIV-infected patients using urine and sputum samples. PLoS One 2010; 5: e9848.

16 Shah M, Variava E, Holmes CB, et al. Diagnostic accuracy of a urine lipoarabinomannan test for tuberculosis in hospitalized patients in a High HIV prevalence setting. J Acquir Immune Defic Syndr 2009; 52: 145-151.

17 Mutetwa R, Boehme C, Dimairo M, et al. Diagnostic accuracy of commercial urinary lipoarabinomannan detection in African tuberculosis suspects and patients. Int J Tuberc Lung Dis 2009; 13: 1253-1259.

18 Reither K, Saathoff E, Jung J, et al. Low sensitivity of a urine LAM-ELISA in the diagnosis of pulmonary tuberculosis. BMC Infect Dis 2009; 9: 141.

19 Nicol MP, Allen V, Workman L, et al. Urine lipoarabinomannan testing for diagnosis of pulmonary tuberculosis in children: a prospective study. Lancet global health 2014; 2: e278-e284.

20 Rachow A, Clowes P, Saathoff E, et al. Increased and expedited case detection by Xpert MTB/RIF assay in childhood tuberculosis: a prospective cohort study. Clin Infect Dis 2012; 54: 1388-1396.

21 Graham SM, Ahmed T, Amanullah F, et al. Evaluation of tuberculosis diagnostics in children: 1. Proposed clinical case definitions for classification of intrathoracic tuberculosis disease. Consensus from an expert panel. J Infect Dis 2012; 205:Suppl. 2, S199-208.

22 Alere. http://alerehiv.com/hiv-comorbidities/procedure/ Date last updated: 2012. Date last accessed: Jan 9, 2015.

23 WHO. WHO Immunological Classification of HIV-related diseases in adults and children. http://wwwwhoint/hiv/ pub/guidelines/HIVstaging150307pdf Date last updated: 2007. Date last accessed: Jan 15, 2015.

24 Wood R, Racow K, Bekker LG, et al. Lipoarabinomannan in urine during tuberculosis treatment: association with host and pathogen factors and mycobacteriuria. BMC Infect Dis 2012; 12: 47.

25 Lawn SD, Kerkhoff AD, Vogt M, et al. Diagnostic accuracy of a low-cost, urine antigen, point-of-care screening assay for HIV-associated pulmonary tuberculosis before antiretroviral therapy: a descriptive study. Lancet Infect Dis 2012; 12: 201-209.

26 Lawn SD, Kerkhoff AD, Vogt M, et al. HIV-associated tuberculosis: relationship between disease severity and the sensitivity of new sputum-based and urine-based diagnostic assays. BMC Med 2013; 11: 231.

27 Kroidl I, Clowes P, Mwakyelu J, et al. Reasons for false-positive lipoarabinomannan ELISA results in a Tanzanian population. Scand J Infect Dis 2014; 46: 144-148. 